\title{
A DIAGRAM NOTATION FOR $N$-MODE ARRAY EQUATIONS
}

\author{
BJØRN K. ALSBERG ${ }^{1}$ \\ ${ }^{1}$ Institute of Biological Sciences, University of Wales, Aberystwyth, Ceredigion SY23 3DA, U.K.
}

\begin{abstract}
SUMMARY
An intuitive and user-friendly notation based on a diagrammatical way to visualize and manipulate $N$-mode array equations is proposed. The diagrams have the appearance of graphs and make some manipulations of equations involving higher-mode arrays easier. (c) 1997 by John Wiley \& Sons, Ltd.
\end{abstract}

Journal of Chemometrics, Vol. 11, 251-266 (1997) (No. of Figures: 21 No. of Tables: 0 No. of Refs: 8)

KEY WORDS $\quad N$-arrays; $N$-mode arrays; multiway arrays; higher-order arrays; multilinear forms; notation; graphs; Feynman diagrams

\section{INTRODUCTION}

Standard vector and matrix notation is very powerful. The typographic formulation captures without difficulty the different ways to manipulate the data objects. Typographically one may regard scalars as zero-dimensional, vectors as one-dimensional and matrices as two-dimensional objects. A similar extension to $\mathrm{N}$-mode arrays will not be successful. Three-mode arrays can of course be described on paper as drawings of boxes, but it is a cumbersome notation which cannot be used efficiently in algebraic manipulations. It has, however, the advantage of rapidly conveying the basic idea of the three-mode data structure. For four-mode or higher-mode arrays the 'drawing notation' quickly collapses. Useful notations therefore do not employ such approaches.

There are some important criteria that should ideally be valid for any efficient $N$-mode notation: it should be unambiguous, useful for complex algebraic manipulations and intuitive at the same time. Unnecessary features introduced by the notation should be kept at a minimum and the underlying data structure should be represented in a logical way. The representation should not change when going from e.g. two to three modes. Such a change happens when, e.g. three-mode arrays are represented as matrices using the vec operator' (this is also referred to as 'unfolding'). A good notation should make it easy to discover symmetries and patterns. In addition, it should be scalable to larger problems, i.e. equations involving a large number of array elements. Most notations in use today do not satisfy all these criteria simultaneously. And, of course, mathematical rigour has preceded the need for intuitive and user-friendly notations.

A type of notation that satisfies all the requirements above is the use of diagrams. Diagrammatical approaches to complex tensor equations have been applied with success to fields such as theoretical physics, ${ }^{2}$ computer graphics ${ }^{3}$ and group theory. ${ }^{4}$ The most famous approach is the Feynman diagram notation $^{2}$ which has been used in many-body problems. It is today a standard tool for manipulating tensor equations explaining interactions between elementary particles. Feynman diagrams include several features related to quantum mechanics which are not necessary for solving $N$-mode array problems in general. The present article thus presents a diagram notation similar (but not equal) to

Correspondence to: Bjørn K. Alsberg, e-mail: bka@aber.ac.uk 
Feynman diagrams which is adapted to $\mathrm{N}$-mode array problems occurring in chemometrics and psychometrics.

\section{BASIC RULES AND PROPERTIES}

The standard use of summation signs and array elements containing indices (e.g. a formula such as $\left.\Sigma_{i} \Sigma_{j} x_{i j k} y_{i p} z_{j}\right)$ is here referred to as the explicit summation notation. The diagram notation is based on a mapping from this standard notation into structures which have the appearance of graphs. ${ }^{5}$ Some graph terminology is therefore appropriate to use here.

The following rules and properties are associated with the diagram notation.

1. A diagram contains nodes and edges. An edge is drawn as a line attached to either one or two nodes. An edge attached to two nodes is said to be connected and an edge attached to one node only is said to be unconnected.

2. A node corresponds to an $N$-mode array element. The edges signify the indices of the array element.

The number of edges is the same as the number of indices in an array. Thus scalars, vectors and matrices have zero, one and two edges respectively. The extension to $N$-mode arrays is logical; see Figure 1.

3. A node is drawn as a cirlce with the name of the array inside. In order to clarify, index names may be introduced in the vicinity of the edges. Often it is necessary to indicate the first index or mode. In such cases the first index is marked with a small bar perpendicular to the edge. This small bar is called the first index pointer or just the fip. The remaining nodes and their indices are labelled counter-clockwise with respect to the fip. The fip together with the counterclockwise orientation uniquely identifies the indices associated with a node.

4. A connected edge is defined to be a summation over one index; see Figure 2.

See Figure 3 for a few examples from vector and matrix algebra.

5. Only the topology of a diagram is important. Rotations, translations and scaling of the nodes and edges are allowed, but mirror reflections are not. A consequence of not allowing mirror reflections of diagrams is that connecting more than two indices between arrays will result in crossing of edges. The crossings themselves have no meaning.

6. The number of unconnected edges of a node or an expression corresponds to the number of modes (indices) of the $\mathrm{N}$-array. This number is hereafter referred to as the mode number, which corresponds to the degree of a node in graph theory. The operator $\mathcal{M}$ applied to an array $\underline{\mathbf{X}}$ returns the mode number of $\underline{\mathbf{X}}$.

The following property holds for all diagrams.

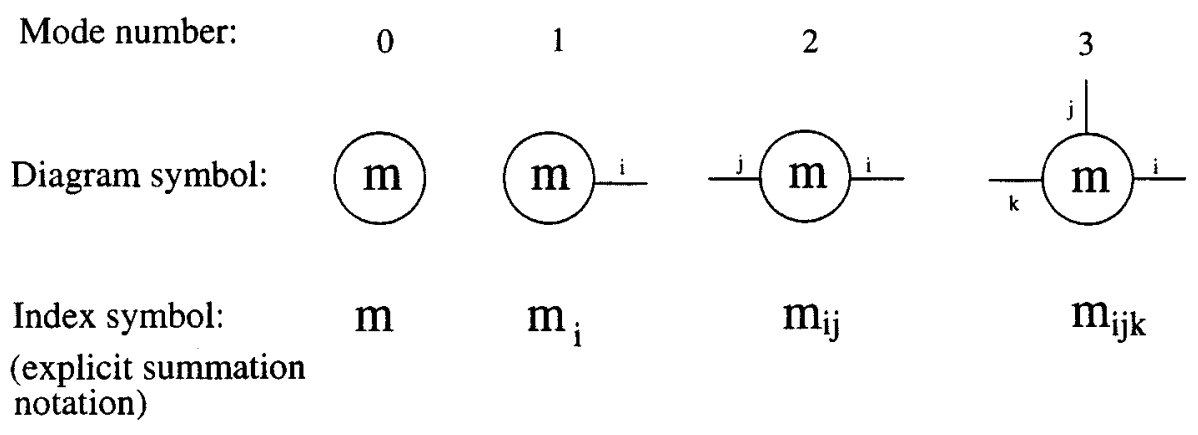

Figure 1. Representations of array elements in the diagram notation and the explicit summation notation 


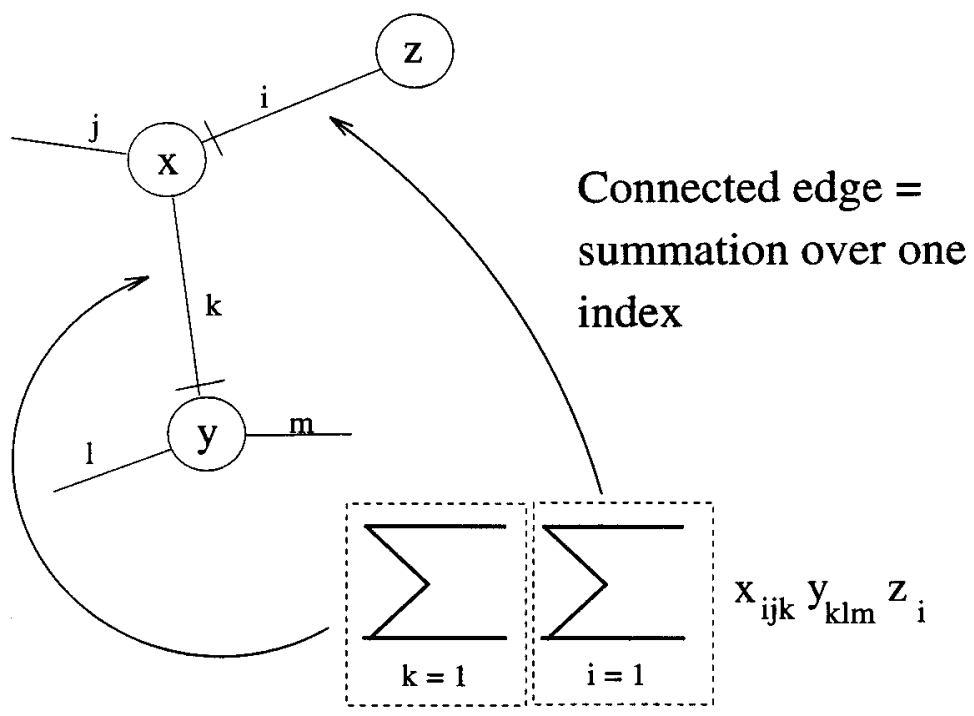

Figure 2. This figure illustrates the main ideas behind the diagram notation

\section{Property 1}

Let a diagram expression of array $\underline{\mathbf{Z}}$ consist of $P$ nodes (array elements). Each node $i$ has $E_{i}$ edges, $i \in[1,2, \ldots, P]$. The number of connected edges is $k$. The total number of unconnected edges (the mode number of $\underline{\mathbf{Z}}$ ) is

$$
M(\underline{\mathbf{Z}})=\sum_{i}^{P} M\left(E_{i}\right)-2 k
$$

A

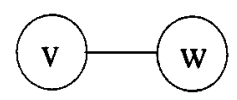

B

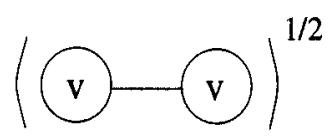

$\mathrm{C}$

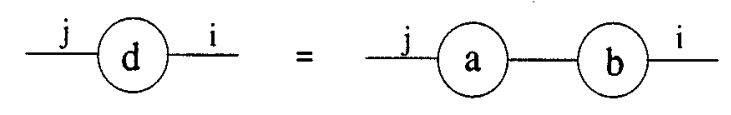

D

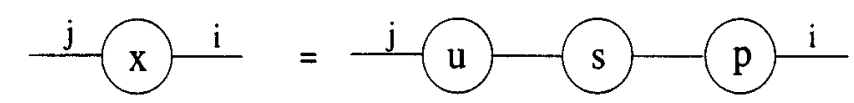

Figure 3. Some examples of standard vector and matrix algebra using the diagram notation: (A) the scalar product $x=\sum_{i=1}^{n} v_{i} w_{i}$; (B) the length of vector $\mathbf{v}$; (C) the matrix product $d_{j i}=\sum_{k=1}^{n} a_{j k} b_{k i}$; (D) the singular value decomposition (SVD) equation for two-mode data arrays $x_{j i}=\Sigma_{k} \Sigma_{l} u_{j k} s_{k l} p_{l i}$ 
For example, an expression of two three-arrays connected by a single edge has a mode number of four (i.e. $3+3-2=4$ ).

\section{NECESSARY EXTENSIONS OF THE NOTATION}

\section{1. $N$-mode Kronecker deltas}

The diagram notation rules stated so far cannot handle cases where the number of array elements involved in a summation of an index is different from two. This is due to the fact that an edge or a line can only connect to two nodes. The problem is solved by using more edges which are connected to a new type of node. This new array contains zeros and ones only. The array used is one version of the $N$-mode Kronecker delta $\left(\delta_{i j k \ldots} \ldots\right)$.

There are two different types of Kronecker deltas used in this notation.

\section{Type $I\left(\underline{\Delta}^{(\mathrm{I})}\right)$}

This is used for summing of an index involving $N \neq 2$ nodes and displaying the trace of an $N$-array. In the diagram notation it is depicted as a filled rectangle. This filled rectangle is also referred to as the sum nexus symbol; see Figures 4(A) and 4(B). The corresponding explicit summation formulae for this figure are

$$
\text { (A) } g_{j}=\sum_{i=1}^{N} a_{i} b_{i} c_{i} x_{i j}
$$

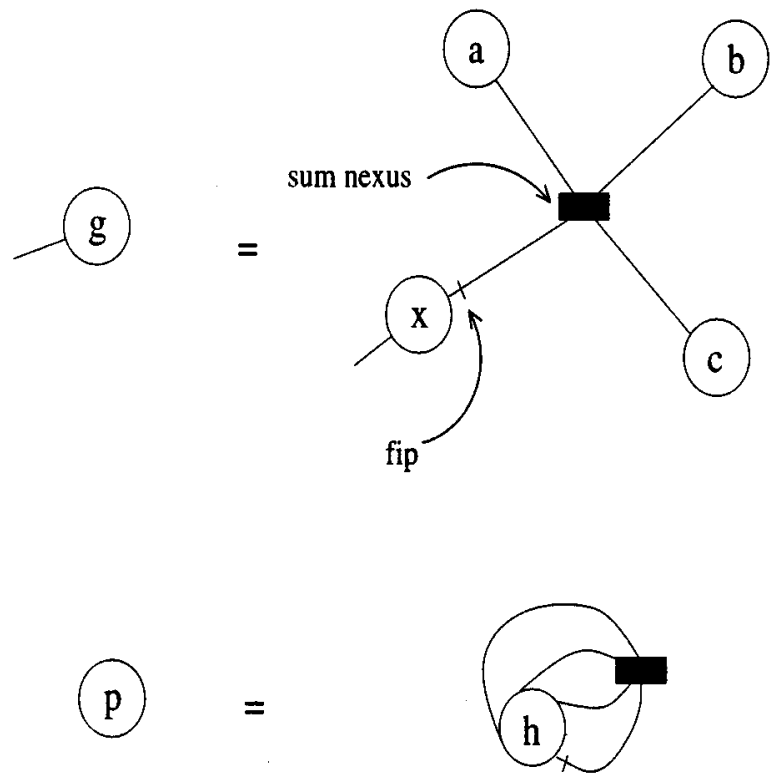

(A)

Figure 4. When more than two arrays are involved in summing of an index, it is necessary to introduce a new symbol which signifies that all the edges belong to the same summation. The sum nexus symbol (a filled rectangle) has therefore been introduced. The sum nexus is a type I Kronecker delta array 
(B) $p=\sum_{i} h_{\text {iiii }}$

When a sum nexus is seen, this means that one and only one index is summed over for all the array elements connected to the sum nexus.

The $N$-mode type I Kronecker delta $\left(\underline{\Delta}^{(\mathrm{I})}\right)$ is generally defined as

$$
\delta_{i_{1} i_{2} \ldots i_{N}}^{(\mathrm{I})}= \begin{cases}1 & \text { if } i_{1}=i_{2}=\cdots=i_{N} \\ 0 & \text { otherwise }\end{cases}
$$

Here $\delta_{i j}$ is the identity matrix. The Kronecker delta for vectors is also defined: $\delta_{i}=1, \forall i$.

Type II $\left(\underline{\Delta}^{(I I)}\right)$

This type is used in array differentiation only and is depicted as a filled circle (the differentiation symbol) of size smaller than the other array nodes. The more detailed use of the differentiation symbol is presented in the section about array differentiation below.

The $N$-mode type II Kronecker delta $\left(\underline{\Delta}^{(I I)}\right)$ is generally defined as

$$
\delta_{i_{1} i_{2} \ldots i_{n} o_{1} o_{2} \ldots o_{n}}^{(I I)}=\left\{\begin{array}{ll}
1 & \text { if } i_{j}=o_{j} \\
0 & \text { otherwise }
\end{array} \forall j \in\{1,2, \ldots, n\}\right.
$$

The $\underline{\Delta}_{N}^{(11)}$ array used in differentiation consists of two sets of indices, $S_{1}=\left\{i_{1}, i_{2}, \ldots, i_{n}\right\}$ and $S_{2}=\left\{o_{1}\right.$, $\left.o_{2}, \ldots, o_{n}\right\} . S_{1}$ is referred to as the set of input indices and $S_{2}$ is referred to as the set of output indices.

\subsection{Chaining}

Sometimes array elements are included in expressions that contain indices not summed over or shared by other array elements. Such nodes cannot be connected to other nodes by edges (summation) or sum nexus symbols. Since no nodes in a diagram expression are allowed to be isolated, some other strategy must be employed. Chaining is here defined to be nodes that touch other nodes but do not connect to them through summation. The perimeters of the nodes coincide in at least one point. Such nodes can in principle be chained to any node in a diagram expression No configuration is preferred to another.

Chaining is important in outer products. An $N$-mode outer product of $N$ vectors $\mathbf{a}, \mathbf{b}, \mathbf{c}, \ldots, \mathbf{q}$ produces an $N$-mode array $\underline{\mathbf{X}}$. It is here defined as

$$
x_{i_{1} i_{2} i_{3} \ldots i_{n}}=a_{i_{1}} b_{i_{2}} c_{i_{3}} \ldots q_{i_{n}}
$$

In the chemometric and psychometric literature it is common to write this as

$$
\underline{\mathbf{X}}=\mathbf{a} \otimes \mathbf{b} \otimes \mathbf{c} \otimes \ldots \otimes \mathbf{q}
$$

Figure 5 shows the principle of node chaining for the outer product between two vectors, e.g. $x_{i j}=v_{i} w_{j}$. The outer product $x_{i j k}=a_{i} b_{j} c_{k}$ is shown in Figure 6 .

It is trivial to extend the chaining to express general array products where each node in the chain is a higher-order mode (i.e. the mode number is more than one) array. 


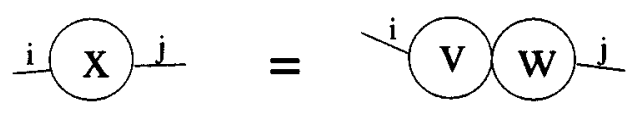

Figure 5. Chaining of nodes is used for expressing outer products in the diagram notation. Here the outer product of two vectors producing a matrix is shown

\section{MANIPULATION OF DIAGRAMS}

\subsection{Solving equations}

When an array expression is to be multiplied with another, we need to specify which index is to be summed over, e.g.

$$
\sum_{i} \sum_{g} a_{e f g} p_{g h i}\left(c_{i j k}+\sum_{m} d_{i m} q_{m j k}\right)=\sum_{g} \sum_{i} a_{e f g} p_{g h i} c_{i j k}+\sum_{g} \sum_{i} \sum_{m} a_{e f g} p_{g h i} d_{i m} q_{m j k}
$$

where $i$ must be specified as the index of summation. This is illustrated in Figure 7 by drawing an additional edge parallel to the existing unconnected edge for the index $i$. It is of course not necessary to do this if only one unconnected edge exists for all the terms. If more than one index needs to be summed over, parallel edges are added to all the necessary unconnected edges. This marking is just temporary and does not have to be maintained during all the steps in a deduction. It is only of interest prior to a multiplication as shown in equation (8) in order to avoid ambiguities in which indices are to be summed.

Diagram equations can be solved and manipulated in very much the same way as matrix equations.

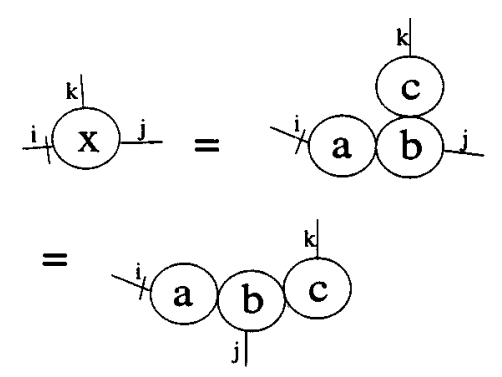

Figure 6. A three-mode vector outer product is shown using chaining of three nodes
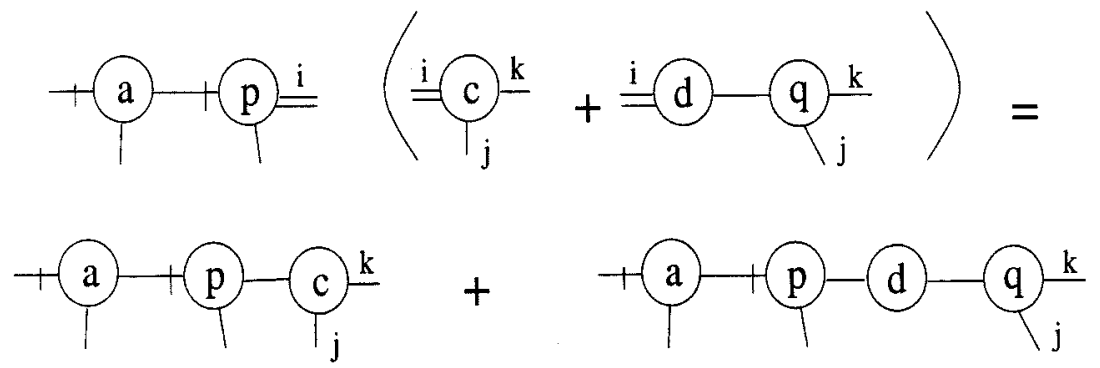

Figure 7. Demonstration of double edges. This is used to signify which edges are to be involved in the final summation. Note that the double edges are no longer presented in the result 

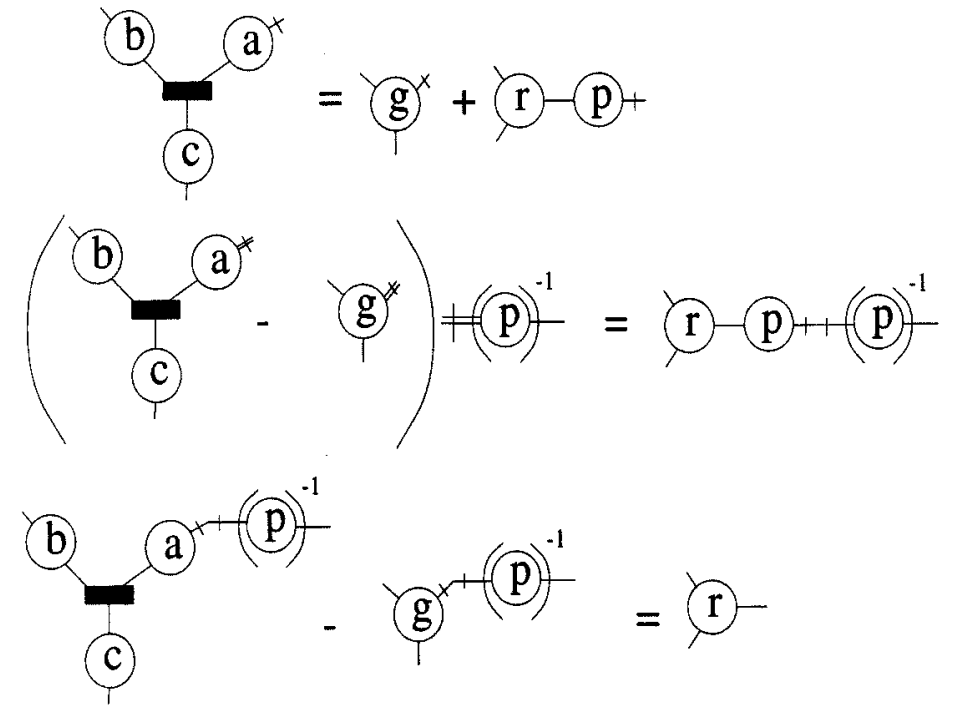

Figure 8. Example of how to solve an array equation. The aim is to write the array $\underline{\mathbf{R}}$ in terms of the other arrays. It is assumed that the matrix $\mathbf{P}$ has an inverse

Those manipulations that are allowed for the explicit summation notation are also valid for the diagram notation. Figure 8 is an example where a three-mode array is equal to the sum of two other three-mode arrays. The matrix $\mathbf{P}$ indicated and multiplied with the three-mode array $\underline{\mathbf{R}}$ is assumed to be invertible. In the middle part of the figure the array $\mathbf{G}$ has been subtracted from the left side and the inverse matrix $\mathbf{P}^{-1}$ is multiplied on both sides. Note the use of double edges which emphasizes which index is to be summed over.

\subsection{Differentiation}

Let $\underline{\mathbf{X}}$ be an $N$-mode array containing $\left[q_{1} \times q_{2} \times \ldots \times q_{N}\right]$ independent variables. Let $G$ be a linear $M$ mode array function which maps $\underline{\mathbf{X}}$ into an $M$-mode array:

$$
G: \mathscr{R}^{q_{1} \times q_{2} \times \ldots \times q_{N}} \rightarrow \mathscr{R}^{p_{1} \times p_{2} \times \ldots \times p_{M}}
$$

The following property holds.

\section{Property 2}

If $\underline{\mathbf{D}}$ is the result of differentiating $G(\underline{\mathbf{X}})$ with respect to $\underline{\mathbf{X}}$, then the mode number of $\underline{\mathbf{D}}$ obeys $\mathcal{M}(\underline{\mathbf{D}})=M(G(\underline{\mathbf{X}}))+M(\underline{\mathbf{X}})$.

Since each element in $\underline{\mathbf{D}}$ is addressed by the indices from both $G(\underline{\mathbf{X}})$ and $\underline{\mathbf{X}}$, the number of indices in $\underline{\mathbf{D}}$ must be the sum of indices in $G(\underline{\mathbf{X}})$ and $\underline{\mathbf{X}}$.

The type II Kronecker delta array is involved in all differentiations where $M(\underline{\mathbf{X}}) \geqslant 1$ and is displayed as the differentiation symbol. When $M(\underline{\mathbf{X}})=1$, both type I and type II Kronecker deltas can be used. We have that

$$
\frac{\partial x_{i_{1} i_{2} \ldots i_{M}}}{\partial x_{j_{1} j_{2} \ldots, j_{M}}}=\delta_{i_{1} i_{2} \ldots i_{M} j_{1} j_{2} \ldots j_{M}}^{(\mathrm{I})}, \quad M \geqslant 1
$$


The steps for performing $N$-mode array differentiation $(N=1,2, \ldots)$ using diagrams are as follows.

1. Replace $\underline{\mathbf{X}}$ with a $2 M(\underline{\mathbf{X}})$-mode $\underline{\Delta}^{(\mathrm{II})}$ array, i.e. if $\underline{\mathbf{X}}$ is a matrix, then $\underline{\Delta}^{(\mathrm{II})}$ has four as mode number.

A minimum of $\mathcal{M}(\underline{\mathbf{X}})$ indices in the differentiation symbol must be free (unconnected). More than $\mathcal{M}(\underline{\mathbf{X}})$ indices can be free in the result if $\underline{\mathbf{X}}$ contained free indices before the differentiation.

The connected edges of the differentiation symbol are by definition the input indices of the $\underline{\Delta}^{(\mathrm{II})}$ array. The fip of $\underline{\mathbf{X}}$ is 'inherited' by the differentiation symbol; it must be such that going counter-clockwise from the fip, the $N$ first edges are the input indices.

2. If $\underline{\mathbf{X}}$ occurs $P$ times in an expression, the result of the differentiation results in a sum of $P$ expressions where each has one $\underline{\Delta}^{(\mathrm{II})}$ array substituted for $\underline{\mathbf{X}}$. This is a result of the chain rule in differentiation.

$N$-mode differentiation is illustrated by two examples. The first is for vector (i.e. $N=1$ ) differentiation:

$$
\begin{aligned}
F\left(x_{q} ; a_{l i j k}\right) & =\sum_{i} \sum_{j} \sum_{k} x_{i} x_{j} x_{k} a_{l i j k} \\
\frac{\partial F}{\partial x_{p}} & =\sum_{i} \sum_{j} \sum_{k}\left(\delta_{i p}\right) x_{j} x_{k} a_{l i j k}+\sum_{i} \sum_{j} \sum_{k} x_{i}\left(\delta_{j p}\right) x_{k} a_{l i j k}+\sum_{i} \sum_{j} \sum_{k} x_{i} x_{j}\left(\delta_{k p}\right) a_{l i j k} \\
& =\sum_{j} \sum_{k} x_{j} x_{k} a_{l p j k}+\sum_{i} \sum_{k} x_{i} x_{k} a_{l i p k}+\sum_{i} \sum_{j} x_{i} x_{j} a_{l i j p}
\end{aligned}
$$

which is illustrated in Figure 9. Here the $\underline{\Delta}^{(\mathrm{II})}$ array is defined as
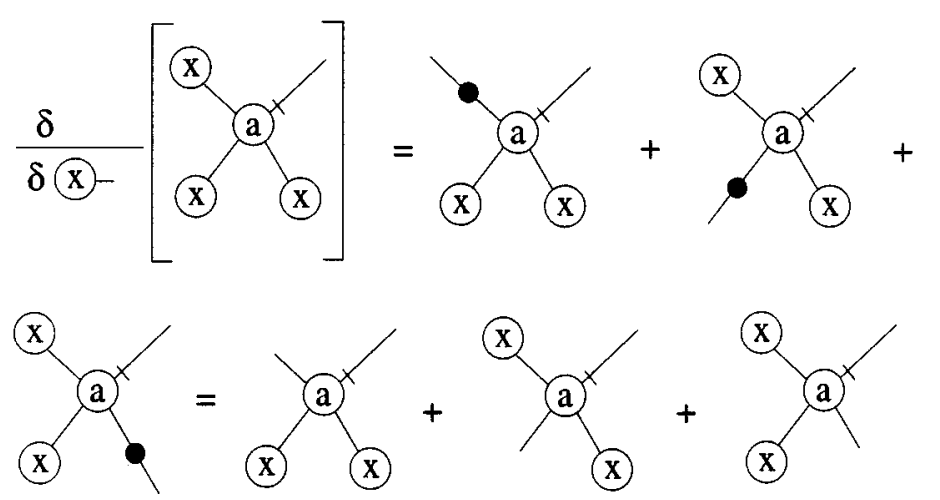

Figure 9. Example which shows the differentiation of an expression involving a three-mode array with respect to the vector $\mathbf{x}$. Three terms are obtained if no symmetry in $\underline{\mathbf{A}}$ is assumed 


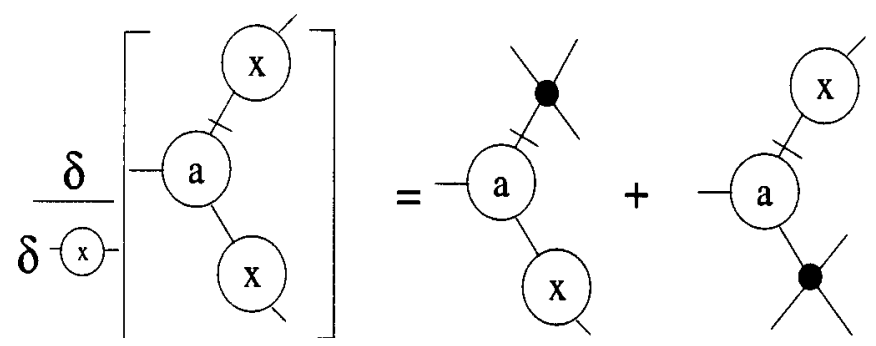

Figure 10. In this example a three-mode array is differentiated with respect to a matrix. In general, when differentiating with respect to an $N$-mode array, we replace that array in the expression with a $2 N$-mode type II Kronecker delta, visualized as a small filled circle (the differentiation symbol). Symmetry in the $\underline{\mathbf{A}}$ array is not assumed

$$
\left(\frac{\partial x_{j}}{\partial x_{k}}\right)=\delta_{j k}
$$

In the next example, differentiation with respect to a matrix $\mathbf{X}$ is performed $(N=2)$ :

$$
\begin{aligned}
G\left(x_{i j}\right) & =\sum_{i} \sum_{k} x_{i p} x_{k q} a_{i j k} \\
\frac{\partial G}{\partial x_{s t}} & =\sum_{i} \sum_{k}\left(\delta_{i p s t}^{(\mathrm{II})}\right) x_{k q} a_{i j k}+\sum_{i} \sum_{k} x_{i p}\left(\delta_{k q s t}^{(\mathrm{II})}\right) a_{i j k}
\end{aligned}
$$

This equation is illustrated in diagram notation in Figure 10.

The type II Kronecker delta can be written as an array outer product of identity matrices using chaining; see Figure 11. It is therefore possible to replace the differentiation symbol by a chain of identity matrices.

This relation is useful for simplification of equations involved in $\mathrm{N}$-mode array differentiation; see Figure 12.

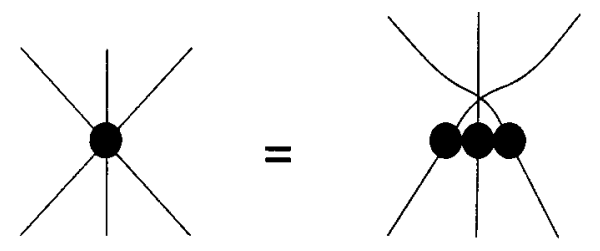

Figure 11. The differentiation symbol can be depicted as a chain of identity matrices. Here a six-mode differentiation symbol is shown 


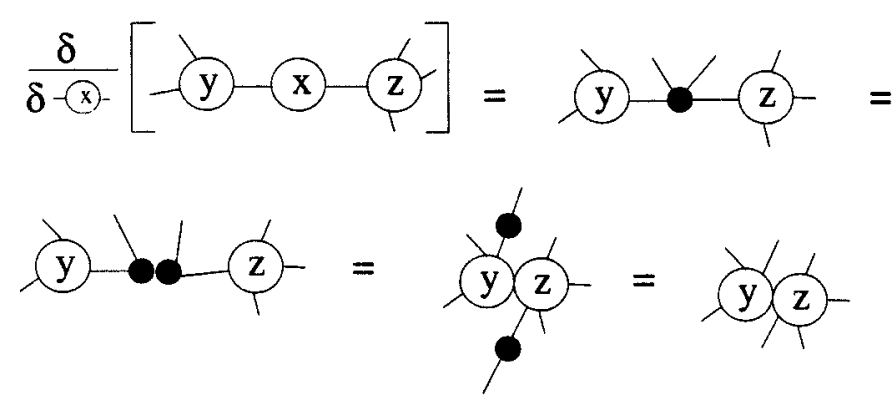

Figure 12. Converting the differentiation symbol to a chain of identity matrices is here demonstrated to be useful for simplifying equations. The result is a chaining of the arrays $\underline{\mathbf{Y}}$ and $\underline{\mathbf{Z}}$. There are no summing or shared indices in the result

\section{EXAMPLES}

\subsection{Differentiation of $N$-mode array traces}

Performing a differentiation of an $N$-mode array trace expression involves replacement of $\underline{\mathbf{X}}$ with a $2 \mu(\underline{\mathbf{X}})$-mode differentiation symbol. After such a substitution the expression is no longer a trace, since at least $N=\mathcal{M}(\underline{\mathbf{X}})$ edges from the $2 N$-mode differentiation symbol are free.

Here the diagram expression of a matrix trace is referred to as a 'ring' and the differentiation symbol is said to 'open' this ring when it substitutes $\mathbf{X}$. The reason why it has the appearance of a ring is that the trace is defined to be the summation over one index used by both modes, i.e.

$$
\operatorname{tr}(\mathbf{M})=\sum_{i} M_{i i}
$$

This means that we only get a ring structure for matrices. For all other higher-mode traces the use of the sum nexus is necessary.

The following examples are presented in diagram form in Figures 13-15:

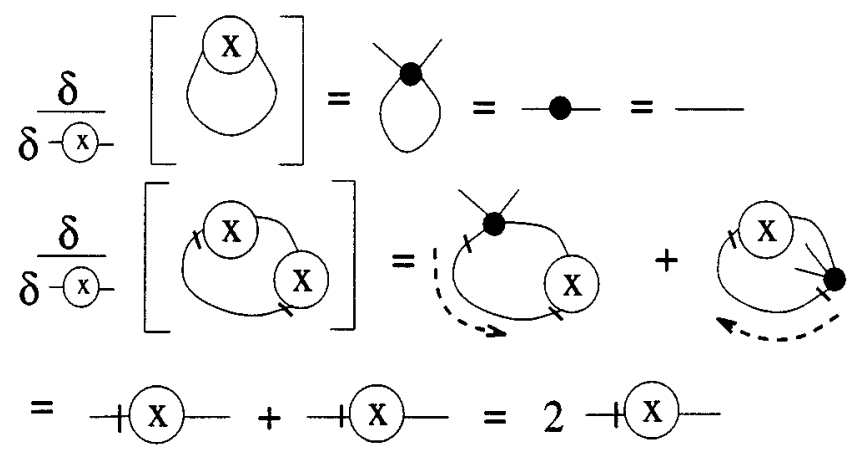

Figure 13. The upper part of the figure shows differentiation of a trace of a matrix with respect to itself. This is equal to the identity matrix. Note that a four-mode $\Delta^{(I I)}$ array is substituted for every occurrence of $\mathbf{X}$ in an expression. In the lower part is the differentiation of $\operatorname{tr}\left(\mathbf{X} \mathbf{X}^{\mathrm{T}}\right)$ with respect to $\mathbf{X}$. Note that broken arrows indicate along which way it is necessary to read in order to obtain the correct transposition of the matrices. The direction of an arrow is determined by the direction from the differentiation symbol to the nearest fip 


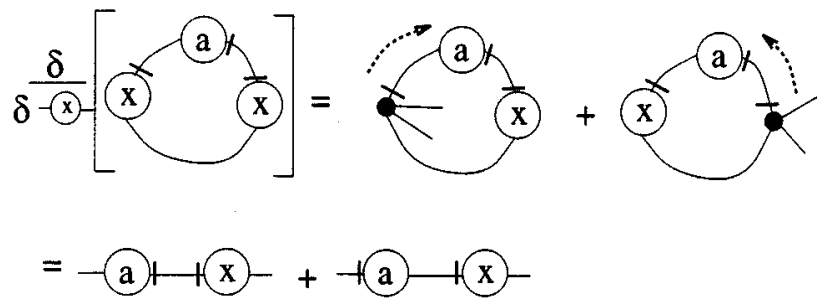

Figure 14. Diagram deduction of $\partial \operatorname{tr}\left(\mathbf{X}^{\mathrm{T}} \mathbf{A} \mathbf{X}\right) / \partial \mathbf{X}$

$$
\begin{gathered}
\frac{\partial \operatorname{tr}(\mathbf{X})}{\partial \mathbf{X}}=\mathbf{I} \\
\frac{\partial \operatorname{tr}\left(\mathbf{X}^{\mathrm{T}} \mathbf{X}\right)}{\partial \mathbf{X}}=2 \mathbf{X} \\
\frac{\partial \operatorname{tr}\left(\mathbf{X}^{\mathrm{T}} \mathbf{A} \mathbf{X}\right)}{\partial \mathbf{X}}=\mathbf{A} \mathbf{X}+\mathbf{A}^{\mathrm{T}} \mathbf{X} \\
\frac{\partial \operatorname{tr}\left(\mathbf{X} \mathbf{A} \mathbf{X}^{\mathrm{T}} \mathbf{B}\right)}{\partial \mathbf{X}}=\mathbf{B}^{\mathrm{T}} \mathbf{X} \mathbf{A}^{\mathrm{T}}+\mathbf{B X} \mathbf{A}
\end{gathered}
$$

It is important to note that the type II Kronecker delta replacing the $\mathbf{X}$ matrix gets (or 'inherits') a fip in a position comparable to $\mathbf{X}$. When the four-mode $\underline{\Delta}^{(\mathrm{II})}$ array opens up the trace ring, the positions of the matrices are read in the direction from the filled circle of the $\underline{\Delta}^{(\mathrm{II})}$ array to the fip along the edge to the next matrix. Arrows are included in Figures 13 and 14 to emphasize the directions indicated by the fips.

Similarly, differentiating with respect to a three-mode array produces a six-mode type II Kronecker array which splits open the $\mathrm{N}$-mode trace. The results for higher-mode differentiation are very similar to what is obtained for differentiation with respect to matrices. Differentiating the trace of a threemode array with respect to itself results in a three-mode type I Kronecker delta; see Figure 16. In Figures 17 and 18, three-mode differentiation with respect to the $\underline{\mathbf{C}}$ array is applied to the error term of the Tucker3 model. The result is the same as that of performing multiplication on both sides by the transposed loading matrices $\mathbf{E}, \mathbf{G}$ and $\mathbf{H}$. This estimate of the core arrays is true only when $\mathbf{E}, \mathbf{G}$ and $\mathbf{H}$ are fully orthonormal.

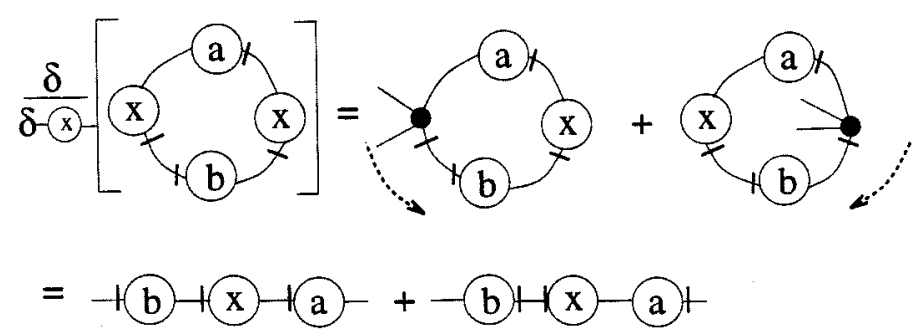

Figure 15. Diagram deduction of $\partial \operatorname{tr}\left(\mathbf{X} \mathbf{A} \mathbf{X}^{\mathrm{T}} \mathbf{B}\right) / \partial \mathbf{X}$ 

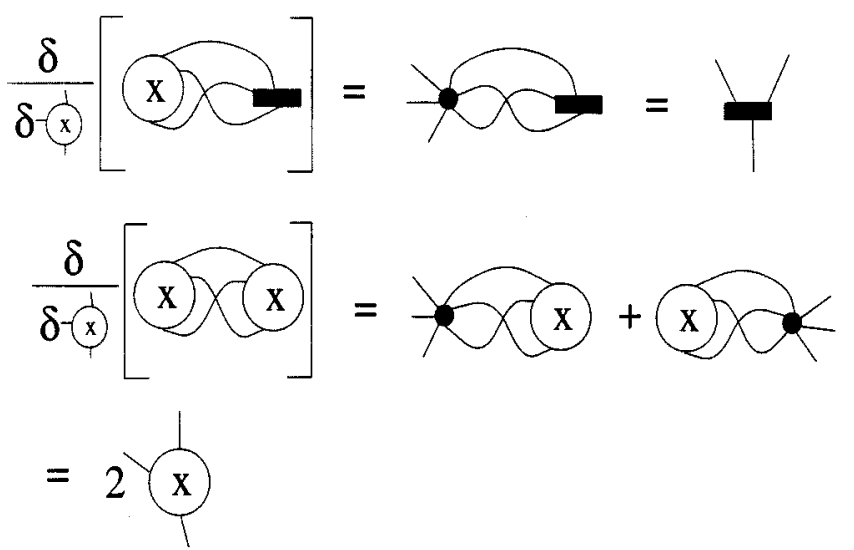

Figure 16. The upper illustration shows differentiation of the trace of a three-mode array which is equal to the three-mode identity array (type I). Below, differentiation of the trace of an expression involving two three-mode $\underline{\mathbf{X}}$ arrays is demonstrated to be the same as $2 \underline{\mathbf{X}}$

\subsection{PARAFAC}

The use of outer products is of importance for expressing the parallel factor analysis (PARAFAC) ${ }^{6}$ model where the core array is restricted to be an identity array (a $\underline{\Delta}^{(\mathrm{I})}$ array). In standard notation, for a three-mode decomposition we get

$$
\underline{\mathbf{X}}=\sum_{i=1}^{n} \mathbf{d}_{i} \otimes \mathbf{e}_{i} \otimes \mathbf{f}_{i}
$$

When using the vec operator to represent three-mode arrays as matrices, the following is obtained:

$$
\mathbf{X}=\mathbf{D I}\left(\mathbf{E}^{\mathrm{T}} \otimes \mathbf{F}^{\mathrm{T}}\right)
$$

where $\mathbf{I}$ is the unfolded three-mode identity array $\Delta^{(\mathrm{I})}$. Often $\mathbf{D}, \mathbf{E}$ and $\mathbf{F}$ are constrained to be suborthonormal loading matrices, but they do not need to be.

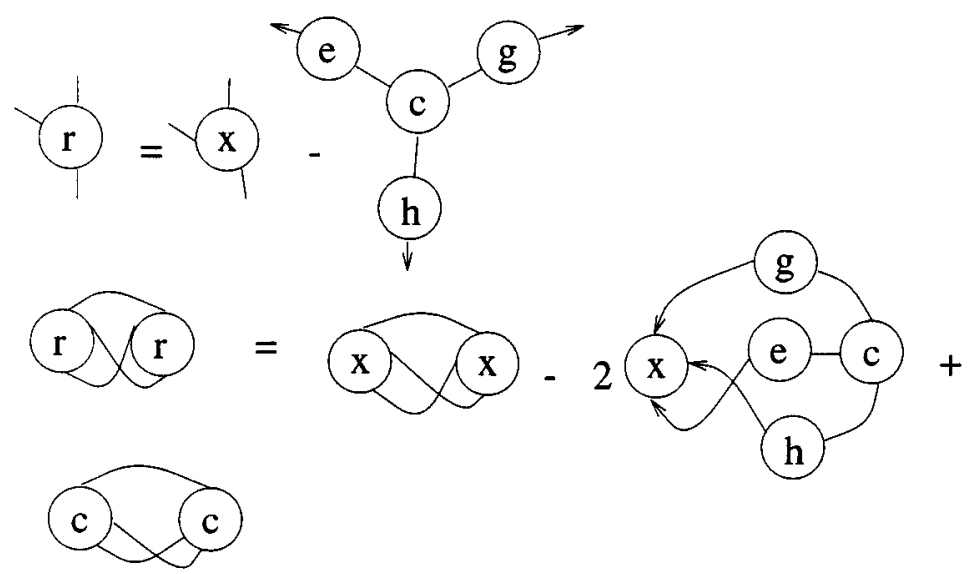

Figure 17. The Tucker 3 model with the error array $\underline{\mathbf{R}}$ is expressed in terms of $\underline{\mathbf{X}}, \underline{\mathbf{C}}$ and the loading matrices. In the lower part of the figure the error scalar value is written in terms of the other array elements 


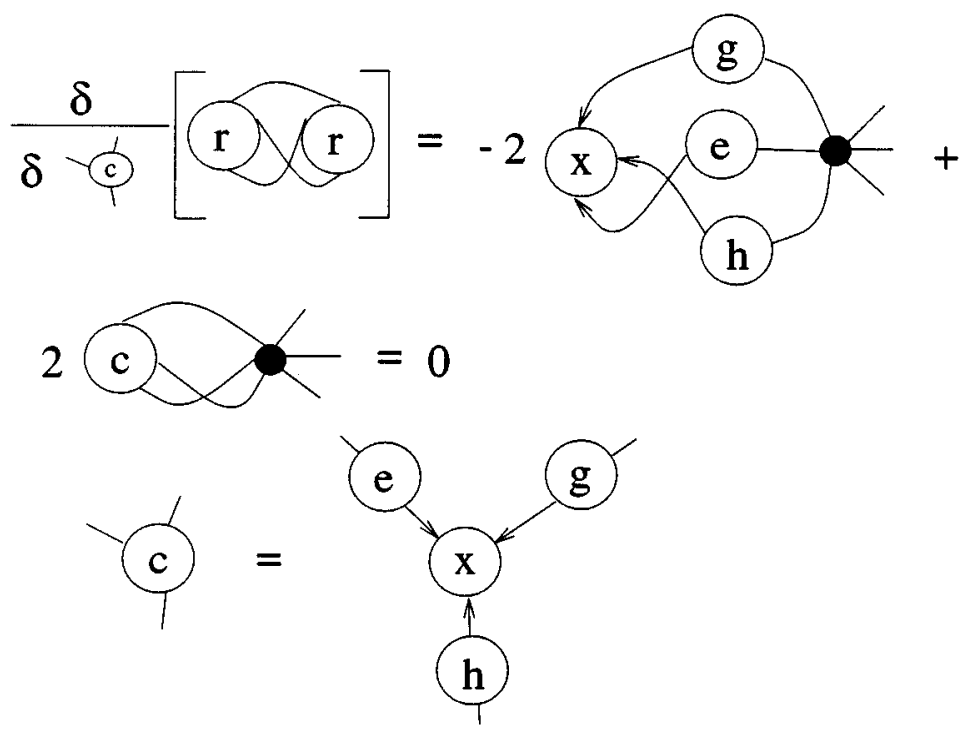

Figure 18. Here the error scalar value in Figure 17 is differentiated with respect to the $\underline{\mathbf{C}}$ array. At each occurrence of the $\underline{\mathbf{C}}$ array it is replaced with the six-mode Kronecker type II array. In the bottom part of the figure the ring opening by the type II Kronecker delta has been performed and one expression has been moved to the other side of the equation sign. This result is the same as if $\underline{\mathbf{X}}$ in Figure 17 had been multiplied by the transposed versions of the loading matrices. The arrows indicate that the matrices are orthonormal

The PARAFAC model for the three-mode case is shown using diagrams in Figure 19. Here chaining has been employed to represent outer products.

\subsection{CANDELINC}

In two-mode CANDELINC (canonical decomposition with linear constraints) ${ }^{7}$ a matrix $\mathbf{Y}$ with dimensions $[J \times K]$ is made from a bilinear model:

Three-mode example
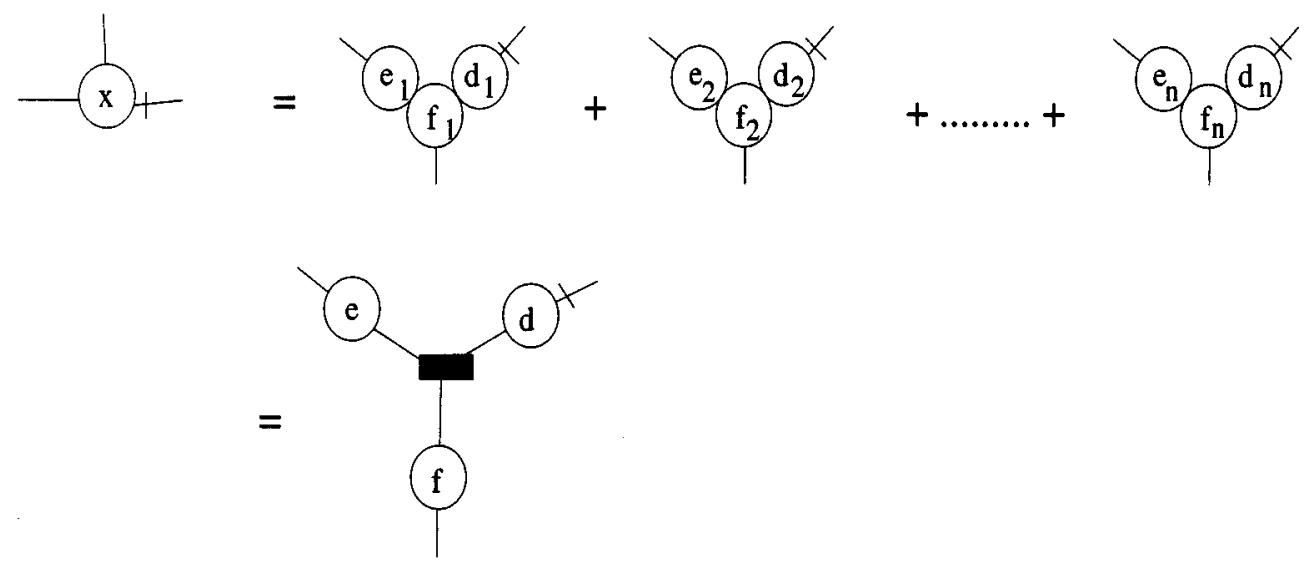

Figure 19. Illustration of trilinear PARAFAC decomposition 


$$
\mathbf{Y} \approx \mathbf{A}_{1} \mathbf{A}_{2}^{\mathrm{T}}
$$

where $\mathbf{A}_{1}$ and $\mathbf{A}_{2}$ contain the parameters to be estimated. These matrices are constrained as follows:

$$
\begin{aligned}
& \mathbf{A}_{1}=\mathbf{X}_{1} \mathbf{T}_{1} \\
& \mathbf{A}_{2}=\mathbf{X}_{2} \mathbf{T}_{2}
\end{aligned}
$$

$\mathbf{X}_{1}$ and $\mathbf{X}_{2}$ are known as 'design matrices' and assumed to have full column rank. $\mathbf{T}_{1}$ and $\mathbf{T}_{2}$ are unknown parameter matrices that must be estimated. The design matrices $\mathbf{X}_{n}$ are suborthonormal:

$$
\mathbf{X}_{n}^{\mathrm{T}} \mathbf{X}_{n}=\mathbf{I}, \quad n \in[1,2]
$$

It can be shown that the problem is solved by finding the matrices $\mathbf{T}_{1}$ and $\mathbf{T}_{2}$ that minimize $N\left(\mathbf{X}_{1}^{\mathrm{T}} \mathbf{Y} \mathbf{X}_{2}-\mathbf{T}_{1} \mathbf{T}_{2}^{\mathrm{T}}\right)$ (for any matrix $\mathbf{Z}, N(\mathbf{Z})=\operatorname{tr}\left(\mathbf{Z} \mathbf{Z}^{\mathrm{T}}\right)$ ). In the two-mode case, $\mathbf{T}_{n}, n \in[1,2]$, is found by using PCA.

For the $N$-mode case $^{7}$ we seek to fit the model

$$
y_{i_{1} i_{2} \ldots i_{N}} \approx \sum_{r=1}^{R} a_{i_{1} r}^{(1)} a_{i_{2} r}^{(2)} \ldots a_{i_{N} r}^{(N)}
$$

where $\approx$ denotes a least squares fit. This formula is depicted using diagrams in Figure 20. The arrows used on the edges in the figure are simply a way to label indices on suborthonormal matrices: when two arrowheads point head-to-head, the result is an identity matrix.

It is assumed that

$$
\mathbf{A}_{n}=\mathbf{X}_{n} \mathbf{T}_{n}, \quad n \in[1,2, \ldots, N]
$$

Following the analogous approach as for the two-mode case, a solution is found by

$$
z_{s_{1} s_{2} \ldots s_{N}} \approx \sum_{r=1}^{R} t_{s_{1} r}^{(1)} t_{s_{2} r}^{(2)} \ldots t_{s_{N} r}^{(N)}
$$

where

$$
z_{s_{1} s_{2} \ldots s_{N}} \equiv \sum_{i_{1}} \sum_{i_{2}} \ldots \sum_{i_{N}} y_{i_{1} i_{2} \ldots i_{N}} x_{i_{1} s_{1}}^{(1)} x_{i_{2} s_{2}}^{(2)} \ldots x_{i_{N} s_{N}}^{(N)}
$$

This equation is illustrated in Figure 21 using the diagram notation. The dots in the figure indicate several additional modes. In this figure the multiplication on both sides of the equation by the suborthonormal matrices $\mathbf{X}_{n}$ is shown. At the right-hand side of the diagram equation the $\mathbf{X}_{n}^{\mathrm{T}} \mathbf{X}_{n}=\mathbf{I}$, $n \in[1,2, \ldots, N]$, occur and the resulting identity arrays are replaced by unconnected edges attached to the $\mathbf{A}_{n}, n \in[1,2, \ldots, N]$, array elements.

\section{DISCUSSION}

The major advantage of the diagram notation is that mathematical manipulation is possible for array equations in general. Diagrams are not just nice presentations of algebraic structures, but symbols that can be manipulated. The diagram notation presents graphically the index topology of complex array equations, which should make it easier for the investigator to detect, e.g. symmetries and interesting 
patterns. Diagrams may thus be particularly suited for teaching purposes as well as when expressing new ideas in $N$-mode algebra.

The diagram notation opens up the possibility of interactive computer tools operating on $\mathrm{N}$-mode array equations. To the author's knowledge there are today no high-level programming tools (languages) available for the investigator who wishes to work efficiently with $N$-mode data array equations and algorithms. For matrix manipulation there are powerful languages such as Matlab and Maple that let the investigator formulate expressions which are similar to standard matrix notation.

Construction of an analogous tool for $N$-mode problems based on diagram notation is possible. Such a program for manipulation of traditional Feynman diagrams has already been constructed ${ }^{8}$ but is of little use to scientists in the fields of chemometrics and psychometrics.

The field of array (and tensor) algbebra is so complex that it may not be realistic to expect that one type of notation will cover all the needs for manipulation and understanding of such equations. The diagram notation should therefore be viewed as supplementary to the already existing notations and not as an attempt to completely replace the other notations. The diagram notation may be an excellent tool for, e.g. introducing new scientists to $\mathrm{N}$-mode array (tensor) algebra and problems related to $\mathrm{N}$ mode index topology. The demand for a more user-friendly notation will increase in proportion to the spread and use of programs and theory based on $N$-mode arrays. The diagram notation may be the answer to such a demand.

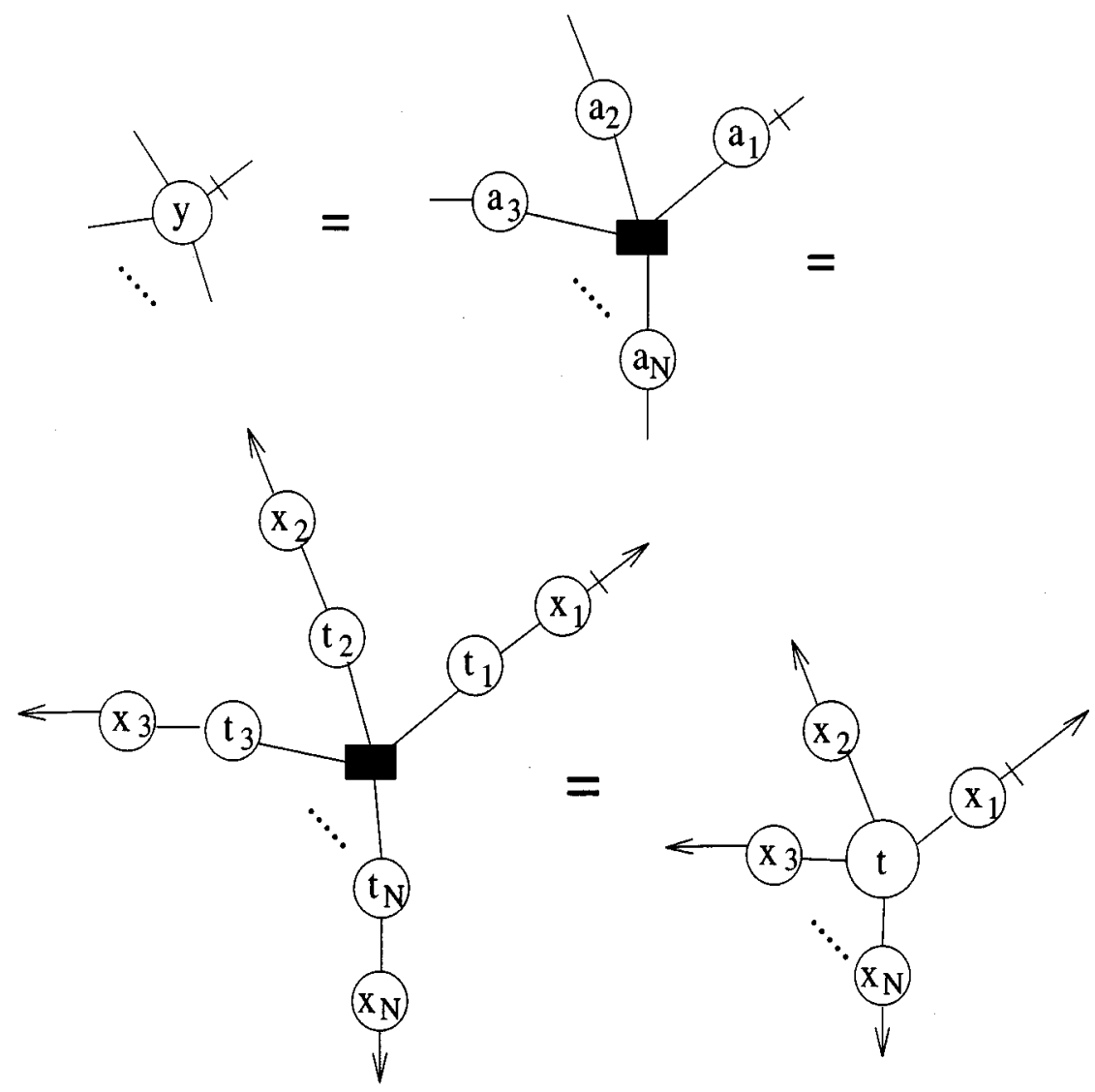

Figure 20. The $N$-mode CANDELINC model. In the lower part of the figure the $\mathbf{A}_{i}$ matrices are replaced by $\mathbf{T}_{i} \mathbf{X}_{i}$. Note that arrows are used on the $\mathbf{X}_{i}$ matrices since they are suborthonormal 


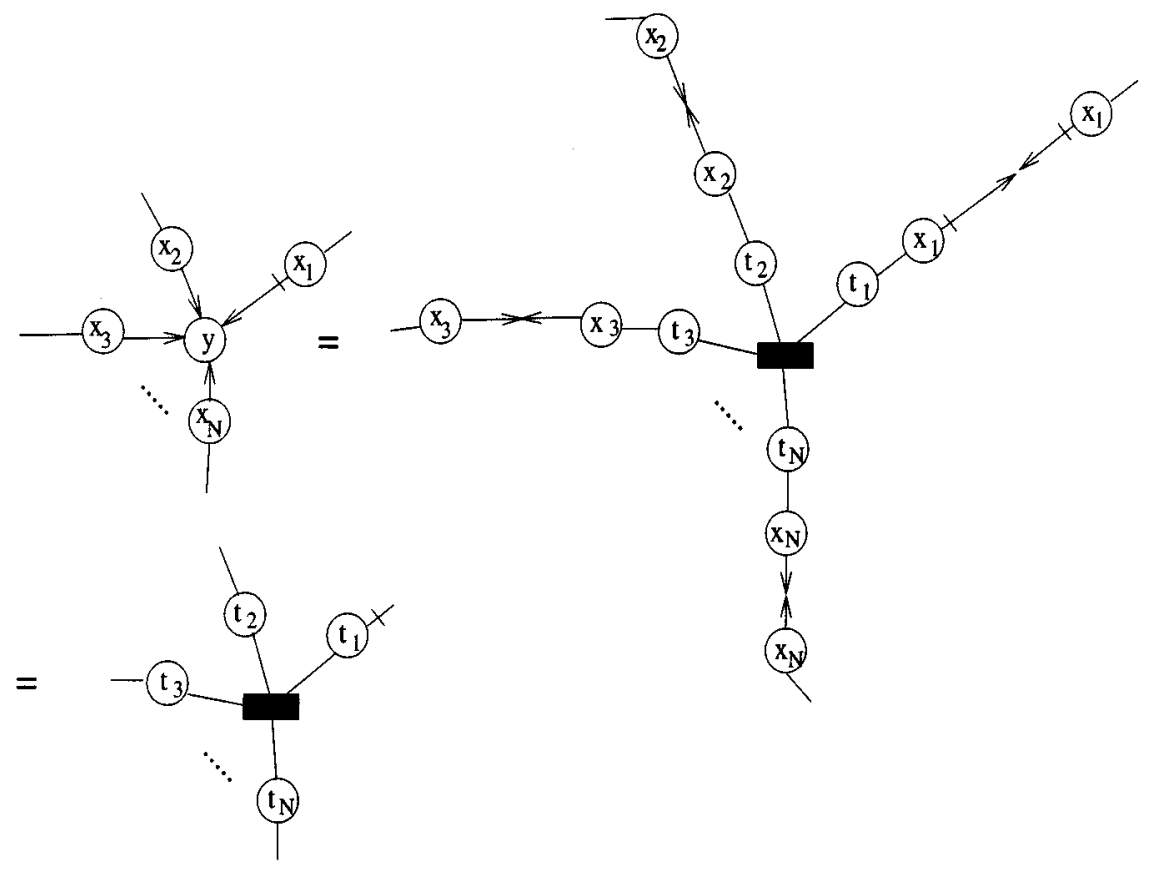

Figure 21. Illustration of how multiplication on both sides of the equation by the transposed $\mathbf{X}_{i}^{\mathrm{T}}$ in $N$-mode CANDELINC will isolate the $\mathbf{T}_{i}$ matrices on the right-hand side of the equation

\section{REFERENCES}

1. J. R. Magnus and H. Neudecker, Matrix Differential Calculus with Applications in Statistics and Econometrics, Wiley, Chichester (1985).

2. R. D. Mattuck, A Guide to Feynman Diagrams in the Many-Body Problem, McGraw-Hill, New York (1976).

3. J. F. Blinn, IEEE Comput. Graph. Appl. 12, 80-85 (1992).

4. G. E. Stedman, Diagram Techniques in Group Theory, Cambridge University Press, Cambridge (1990).

5. J. R. Wilson, Introduction to Graph Theory, 3rd edn, Longman, New York (1985).

6. R. A. Harshman, UCLA Working Papers Phonet. 16, 1-84 (1970).

7. J. D. Carroll, S. Pruzansky and J. B. Kruskal, Psychometrika, 45, 3-24 (1980).

8. J. Kübleck, Böhm and A. Denner, Comput. Phys. Commun. 60, 165-180 (1990). 